\title{
DIÁLOGOS ENTRE ESPIRITUALIDADE E ENFERMAGEM: UMA REVISÃO INTEGRATIVA DA LITERATURA
}

Caren Camargo do Espírito Santoํㅜ, Antonio Marcos Tosoli Gomes², Denize Cristina de Oliveira ${ }^{3}$, Ana Paula Munhen de Pontes ${ }^{4}$, Érick Igor dos Santos ${ }^{5}$, Camila Perroni Marouço da Costa ${ }^{6}$

RESUMO: Esta revisão integrativa objetivou identificar e compreender como a espiritualidade tem sido abordada nas publicações realizadas por enfermeiros e foi realizada nas bases de dados Scopus, Wiley Online Librarye Scientific Eletronic Library Online, usando como palavra-chave espiritualidade. Originaram-se as temáticas: A vivência da espiritualidade em situações de saúde e doença; A espiritualidade e o profissional de saúde; e A espiritualidade vivida por cuidadores e familiares. A espiritualidade está associada à busca de sentido na vida no enfrentamento da dor. Destaca-se a importância do desenvolvimento da espiritualidade no cuidado e conclui-se que a maioria das publicações de enfermagem não se refere, especificamente, à espiritualidade, mas esta temática é encontrada no conjunto dos resultados.

DESCRITORES: Espiritualidade; Enfermeiros; Cuidados de enfermagem.

\section{DIALOGS BETWEEN SPIRITUALITY AND NURSING: AN INTEGRATIVE LITERATURE REVIEW}

\begin{abstract}
This integrative review aimed to identify and investigate how spirituality has been addressed in publications produced by nurses. It was made using the databases Scopus, Wiley Online Library, and Scientific Electronic Library Online, using 'spirituality' as the key-word. The following themes were produced: The experience of spirituality in situations of care and illness; Spirituality and the health professional; and Spirituality experienced by caregivers and family members. Spirituality is associated with the search for meaning in life in coping with pain. The importance of developing spirituality in care is stressed, and it is concluded that the majority of nursing publications do not refer, specifically, to spirituality, but that this issue is found in the set of the results.
\end{abstract}

DESCRIPTORS: Spirituality; Nurses; Nursing care.

\section{DIÁLOGOS ENTRE ESPIRITUALIDAD Y ENFERMERÍA: UNA REVISIÓN INTEGRATIVA DE LA LITERATURA}

RESUMEN: Esta revisión integrativa tuvo la finalidad de identificar y comprender cómo la espiritualidad viene siendo abordada en las publicaciones realizadas por enfermeros, siendo realizada en las bases de datos Scopus, Wiley Online Library y Scientific Eletronic Library Online, usando como palabra clave espiritualidad. Resultaron las temáticas: La vivencia de la espiritualidad en situaciones de salud y enfermedad; La espiritualidad y el profesional de salud; y La espiritualidad vivida por cuidadores y familiares. La espiritualidad está asociada a la búsqueda de sentido en la vida cuando en un estado de dolor. Se destaca la importancia del desarrollo de la espiritualidad en el cuidado y se concluye que la maioría de las publicaciones de enfermería no se refiere, específicamente, a la espiritualidad, pero esta temática es encontrada en el conjunto de los resultados. DESCRIPTORES: Espiritualidad; Enfermeros; Cuidados de enfermería.

\footnotetext{
${ }^{1}$ Enfermeira. Mestre em Enfermagem. Doutoranda pelo Programa de Pós-Graduação em Enfermagem da Universidade do Estado do Rio de Janeiro - UERJ. Professora substituta da Faculdade de Enfermagem do Estado do Rio de Janeiro.

${ }^{2}$ Enfermeiro. Doutor em Enfermagem. Professor do Departamento de Enfermagem Médico-Cirúrgica da Faculdade de Enfermagem daUERJ. ${ }^{3}$ Enfermeira. Doutora em SaúdePública. Professora do Departamento de Fundamentos de Enfermagem da Faculdade deEnfermagem da UERJ. ${ }^{4}$ Enfermeira. Mestre em Enfermagem. Doutoranda pelo Programa de Pós-Graduação em Enfermagem da UERJ.

${ }^{5}$ Enfermeiro. Mestre em Enfermagem.

${ }^{6}$ Enfermeira. Mestranda em Enfermagem pelo Programa de Pós-Graduação em Enfermagem da UERJ.
}

Autor correspondente:

Caren Camargo do Espírito Santo

Universidade do Estado do Rio de Janeiro

Rua Salomão Filho, 577 - 21331-660 - Rio de Janeiro-RJ-Brasil

E-mail: carencamargo.enf@gmail.com
Recebido: 11/09/2012 Aprovado: 17/01/2013 


\section{INTRODUÇÃO}

A espiritualidade é uma temática que está sendo muito discutida atualmente no campo científico, principalmente na área da saúde. Inúmeros estudos estão sendo desenvolvidos relacionando a espiritualidade com a qualidade de vida, bem como com o enfrentamento de doenças e a promoção e a reabilitação da saúde. A atenção voltada para a dimensão espiritual torna-se cada vez mais necessária à prática assistencial à saúde. A ciência, aos poucos, vem reconhecendo o papel fundamental da espiritualidade na dimensão do ser humano já que ser humano é buscar significado e razão em tudo que está em si mesmo e à sua volta, estando sempre em busca de ser completo e preenchido nas diversas áreas de sua vida ${ }^{(1)}$.

A crise existencial trazida pela doença pode levar o indivíduo a grandes questionamentos sobre sua vida dotados de grande emoção ${ }^{(2)}$. Destaca-se, portanto, a força da espiritualidade como instrumento de promoção da saúde e compreensão no modo de lidar com e vivenciar a doença, na medida em que se relaciona, na maioria das vezes, com dimensões menos tangíveis da complexa estrutura do ser humano que, ao mesmo tempo, tendem a ultrapassar os limites da razão científica, onde, de um modo geral, é conferido significado à vida e, simultaneamente, habitam os valores, as motivações profundas e os sentidos últimos da existência individual e coletiva ${ }^{(3)}$.

Neste contexto, este estudo tem como objetivos identificar como a espiritualidade tem sido abordada nas publicações realizadas por enfermeiros e caracterizá-la de acordo com as temáticas desenvolvidas nos estudos analisados. Sua realização se justifica pela importância que a espiritualidade tem assumido nas discussões científicas na área da saúde, pretendendo-se fornecer um panorama das publicações existentes sobre o tema; torna-se relevante por contribuir com novas reflexões e aprimoramento de saberes sobre a dimensão espiritual do cuidado em saúde e de enfermagem, de uma maneira mais específica.

\section{MÉTODO}

Trata-se de uma revisão integrativa de literatura realizada $^{(4)}$, qualitativa e de natureza descritiva. Para que a revisão integrativa possa ser elaborada faz-se necessário percorrer seis etapas distintas ${ }^{(4)}$. Na primeira etapa, foi definida como questão norteadora: como a espiritualidade é caracterizada através de artigos publicados por enfermeiros em periódicos nacionais e internacionais? Como palavra-chave foi selecionada a palavra espiritualidade.

Na segunda etapa, os critérios de inclusão e exclusão foram estabelecidos e iniciou-se a busca nas bases de dados para seleção dos estudos. A busca foi realizada nos meses de janeiro de 2011 a dezembro de 2012, no portal da CAPES, na subárea Enfermagem, nas seguintes bases de dados: Scopus, Wiley Online Library e Scientific Eletronic Library Online (SciELO). Foram selecionados os artigos publicados em português, inglês ou espanhol, que possuíssem o termo espiritualidade em seus resumos, com texto completo disponível online, e cujos autores deveriam ter a graduação em Enfermagem. Ressalta-se que os artigos com mais de um autor, no qual pelo menos um deles é enfermeiro, também foi incluído na análise. Sendo assim, foram excluídos artigos cujos textos completos não estavam disponíveis online e resultados não apresentavam discussão acerca da espiritualidade.

A terceira etapa da revisão integrativa de literatura foram definidas as informações a serem extraídas. Para a coleta dos dados foi utilizado um instrumento, construído pelos autores, contendo as seguintes variáveis: periódico, idioma, título, autores, tipo de estudo, local do estudo, ano de publicação e principais resultados relacionados à espiritualidade.

A quarta e a quinta etapas foram desenvolvidas ao longo do corpo textual. A quarta etapa correspondeu à fase de análise, avaliação, inclusão e exclusão dos estudos através de uma análise crítica daqueles selecionados. Já durante a quinta etapa ocorre a interpretação e discussão dos resultados encontrados. A sexta e última etapa consistiu na elaboração do documento que contempla a descrição das etapas percorridas pelo revisor e os principais resultados evidenciados da análise dos artigos incluídos.

\section{RESULTADOS}

Como resultado da aplicação dos termos de busca, foram encontrados 337 artigos. Após a aplicação dos critérios de inclusão e exclusão, 50 textos foram selecionados para análise. Destes, um artigo encontrava-se no idioma espanhol, 11 em inglês e os demais em português.

A distribuição das informações referentes à quantidade de artigos publicados por ano de publicação e periódico mostra que em 2008 houve um aumento na produção de artigos, sendo publicados 18\% (09) dos trabalhos, dos quais 8\% (04) foram na Revista Latino-Americana de Enfermagem. Em 2009 obteve-se um 
total de 20\% (10) de artigos, com destaque para as Revistas Escola de Enfermagem da USP e Latino-Americana de Enfermagem, cada uma com 4\% (02) de artigos publicados. Em 2010 houve 16\% (08) de publicações, sendo 4\% (02) na Revista Latino-Americana de Enfermagem. Em 2011 houve 12\% (06) de publicações, com 4\% (02) tanto na Revista Escola de Enfermagem da USP como na Latino-Americana de Enfermagem. Por fim, em 2012, o maior período de publicação juntamente com o ano de 2009, houve 20\% (10) de publicações, com 4\% (02) na Revista Escola de Enfermagem da USP.

Percebe-se que o tipo de metodologia mais utilizado nos estudos é o qualitativo, abarcando 56\% (28) do total de artigos analisados. Após, segue a metodologia quantitativa, com 24\% (12). Quanto ao local de publicação dos estudos originais, nota-se que as pesquisas concentraram-se nas regiões Sudeste, com 43\% (18) e Sul, com 21,4\% (09), totalizando 64,4\% (27). Em seguida, tem-se a região Nordeste, com 9,5\% (04), e o Centro-Oeste, com 7,1\% (03). Nota-se que 19\% (08) foram realizados fora do Brasil.

Os resultados discursivos foram sistematizados, integrados e apresentados a partir de três temáticas: 1) A vivência da espiritualidade em situações de saúde e doença; 2) A espiritualidade e o profissional de saúde; 3) A espiritualidade vivida por cuidadores e familiares.

\section{A vivência da espiritualidade em situações de saúde e doença}

Esta temática é a maior da análise, da qual fizeram parte 24 artigos. Foi discutida a vivência da espiritualidade pelos próprios doentes, bem como por indivíduos sadios. Para alguns indivíduos, a saúde está associada, dentre outros fatores, a ter fé e à religião ${ }^{(5)}$. É discutida a existência de duas dimensões espirituais: a vertical, associada a uma relação com um ser superior e divino; e a horizontal, associada à um sentido maior e à um significado de vida decorrentes de uma relação consigo próprio, com os outros e com o ambiente ${ }^{(6)}$.

Adultos jovens reconhecem a espiritualidade como uma dimensão em suas vidas e muitos manifestam sua crença no divino. O sentido e o significado da vida podem ser encontrados através do relacionamento $\operatorname{com~Deus}^{(7)}$. Os estudos com idosos revelam uma forte vivência espiritual e religiosa, mesmo em situações de hospitalização, onde expressam o cuidado de si através da fé religiosa e do ato de rezar ${ }^{(8)}$. Inclusive, determinados autores apontam que uma das condições associadas ao envelhecimento bem-sucedido é a crença religiosa ou espiritual ${ }^{(9)}$.

Estudos discutem a espiritualidade relacionada à morte ${ }^{(10,13)}$. As crenças e os valores exercem grande influência no processo de morte e morrer. Sendo assim, o momento de luto, o qual constitui um momento de intensa dor, constitui um objeto de ação da espiritualidade ${ }^{(12-13)}$. Pesquisas na área da espiritualidade têm sido desenvolvidas com o apoio de instrumentos como escalas de bem estar espiritual e de qualidade de vida, a qual também abarca a dimensão espiritual. Estudos referentes à aplicação da escala de qualidade de vida de pessoas com a saúde prejudicada revelam que a espiritualidade é estatisticamente significativa, aparecendo como um domínio que possui um dos maiores scores médios ${ }^{(14-17)}$.

Percebe-se, também, que o exercício da espiritualidade pode ser um grande aliado no que se refere ao processo de reabilitação quanto ao abuso e dependência de álcool e outras drogas ${ }^{(18-20)}$. Em pessoas com doenças graves, como câncer, doença renal em fase terminal e HIV/Aids, a espiritualidade e a religiosidade servem como forma de enfrentamento e adesão ao tratamento ${ }^{(21-24)}$. Destaca-se que embora exista o diagnóstico de enfermagem associada à angústia espiritual, segundo a North American Nursing Diagnosis Association International (NANDA I), apenas dois estudos fizeram referência a esta temática ${ }^{(25-26)}$.

Nota-se que outros elementos associados à espiritualidade são a força e a motivação, a esperança e as relações afetivas associadas ao encontro de sentido ${ }^{(27)}$. Outra característica da espiritualidade é o amar e o ser amado também ${ }^{(28)}$. Destaca-se, assim, que o encontro de sentido mobiliza as forças para se continuar vivendo e, neste sentido, este encontro se dá principalmente através dos relacionamentos consigo, com o outro e com o divino.

\section{A espiritualidade e o profissional de saúde}

Nesta temática foram incluídos 16 artigos e diz respeito à inclusão da espiritualidade do profissional de saúde e, especificamente, do enfermeiro no ensino de graduação, no modo de ver as pessoas sob seus cuidados, na assistência direta ao paciente e em sua própria vida. Destaca-se a importância do reconhecimento da dimensão espiritual no processo comunicacional do paciente com o profissional de saúde ${ }^{(29)}$. Ainda assim, os profissionais revelam barreiras para lidar com a espiritualidade do paciente, tais como medo de ofender e de impor suas próprias crenças, de lidar com religiões 
divergentes as deles e a falta de tempo ${ }^{(30-32)}$. Algumas formas de lidar com a espiritualidade são descritas como escuta ativa, a presença e a compaixão(33).

Avaliando-se a qualidade de vida de profissionais de saúde que trabalham em UTI neonatal e pediátrica, médicos e enfermeiros, no que se refere ao domínio espiritualidade/religiosidade/crenças pessoais, apresentaram scores significativos, o que pode demonstrar um melhor maneira de lidar com o estresse cotidiano na atuação profissional ${ }^{(34)}$. Em relação ao ensino desta dimensão na graduação em enfermagem, destaca-se a sua importância e que sua implementação pode se dar a partir de reflexões junto ao aluno sobre o que é espiritualidade e sua importância para o ser humano, identificando, ao mesmo tempo, suas crenças e valores sobre o assunto, além de ministrar aulas teóricas acerca das diferentes religiões ${ }^{(35)}$. Destaca-se a influência da religiosidade no exercício da profissão de enfermagem, sendo a dimensão ético-moral-espiritual um ideal moral da enfermagem ${ }^{(36-37)}$.

Em relação ao profissional enfermeiro, diversos estudos têm tratado de sua espiritualidade sob diferentes perspectivas, como o cuidado de si, a forma de enfrentamento do cotidiano de trabalho e a partir do olhar ao paciente. Constatou-se que os enfermeiros consideram muito importante o apoio espiritual dado ao paciente em processo de morrer e morte ${ }^{(38)}$. Integrando-se a dimensão espiritual ao trabalho cotidiano, este profissional faz com que haja contribuição à melhor qualidade de vida, tanto pessoal quanto profissional ${ }^{(39)}$. Já em outro estudo foi relatado que, para lidar com a tensão do trabalho, técnicos e auxiliares de enfermagem buscavam, na espiritualidade, o enfrentamento do estresse, em contraposição aos enfermeiros, que realizavam atividade física ou conversavam com os outros colegas de trabalho ${ }^{(40)}$. Outro estudo verificou que a saúde espiritual de enfermeiras que não sofriam depressão era melhor do que a das que sofriam ${ }^{(41)}$.

Outras formas de cuidado de si relacionadas à espiritualidade que apareceram nas publicações foram práticas cotidianas de oração, contato íntimo com a natureza e de conexão com uma força superior ${ }^{(42)}$. Destaca-se, portanto, a necessidade de inclusão de temas relacionados à espiritualidade nos currículos de graduação, pois parece que os profissionais ignoram a avaliação espiritual ou apresentam dificuldades de integrar esse cuidado na sua prática ${ }^{(43)}$. Este fato pode ser devido à hegemonia do modelo biomédico no qual os profissionais de saúde estão inseridos, que, dando ênfase à fisiopatologia da doença, pouco valoriza as dimensões subjetivas do ser. Outro fator pode estar relacionado à ausência de disciplinas acadêmicas que abarquem assuntos relacionados à espiritualidade durante a formação profissional, fazendo com que os profissionais sintam-se despreparados para lidar com a demanda do cuidado espiritual. Por fim, deve-se destacar que a dimensão espiritual mostra-se, ainda, não apreensível pelos métodos objetivos da ciência contemporânea ocidental, o que dificulta a sua inserção nas instituições de ensino e de assistência à saúde.

\section{A espiritualidade vivida por cuidadores e familiares}

A espiritualidade vivida por familiares e cuidadores se apresenta, de certa forma, transversal às categorias anteriores, sendo abordada por 10 artigos. As práticas espirituais e religiosas servem não apenas como suporte nas situações enfrentadas, mas abre uma possibilidade para o diálogo sobre a espiritualidade no ambiente hospitalar. Foi possível, também, refletir que, em situações de morte, a dimensão religiosa do familiar contribui para a elaboração do luto e a aceitação deste contexto, bem como de sua própria finitude, diminuindo a angústia e a ansiedade ${ }^{(44)}$.

Um estudo revelou que as mães de jovens assassinados destacaram a crença no divino como fortaleza para sobreviver à morte. Assim, a fé e a própria religião tornam tolerável o insuportável, oferecendo força para lidar com a tragédia ${ }^{(4)}$. O familiar, diante do adoecimento de um ente, passa a conviver com situações de sofrimento e desequilíbrio e é neste sentido que a espiritualidade emerge, dando suporte para o enfrentamento do problema ${ }^{(44-47)}$. Mesmo sendo difícil cuidar de um familiar doente, o cuidado passa a ser parte da dimensão espiritual, dando a sensação de conformidade com as mudanças que o cuidado lhe propicia ${ }^{(46)}$. Além disso, este cuidado, mesmo apreendido como sobrecarregado, gera no cuidador um sentimento de estar cumprindo com sua obrigação, tornando-o gratificante ${ }^{(48)}$.

As crenças existenciais, espirituais ou religiosas, contribuem para que os eventos da vida sejam interpretados positivamente e enfrentados de forma mais eficaz. Ao participar de uma comunidade religiosa, os cuidadores passam a receber variadas formas de apoio, sejam elas espirituais, emocionais e sociais ${ }^{(49)}$. Um dos estudos com familiares de crianças com doença renal crônica destacou que a fé no poder divino é tão grande em alguns cuidadores que acreditam no processo de cura do filho ${ }^{(50)}$. Evidenciou-se, também, que acreditam que Deus pode curar as crianças doentes e protegê-las de complicações clínicas ${ }^{(51)}$. 
Em relação às práticas espirituais, uma que merece destaque é a oração. Mesmo quando não plenamente atendidos em suas preces, os cuidadores são gratos pelas graças concedidas por Deus ${ }^{(51-52)}$. Um estudo que utilizou o conceito de Tríade Trágica, a saber, culpa, sofrimento e morte, verificou que, para o familiar de um paciente crítico, encontrar o sentido de vida frente à Tríade Trágica é perceber o otimismo trágico, como possibilidade de responder às questões da vida de modo positivo e responsável, através de forças espirituais ${ }^{(53)}$. Estas forças traduzem-se em fé, esperança e amor, além de, em algumas situações, em práticas religiosas, que transformam o sofrimento em uma atitude positiva, dando ao familiar um sentido último para a vida. Portanto, pode-se perceber que as práticas, os sentimentos e os valores do cuidador e do familiar são influenciadas por sua espiritualidade e, sendo assim, o processo saúde-doença é vivido, representado e significado também através dela.

\section{CONCLUSÕES}

Verificou-se que a maioria das publicações de enfermagem não se refere especificamente à espiritualidade, mas esta temática é encontrada no conjunto de resultados revelados nos estudos de forma transversal à discussão relacionada ao cuidado. Isto comprova a importância da espiritualidade que, mesmo não sendo o foco do estudo, e, portanto, sem uma investigação direcionada a ela, emerge com grande força.

Constata-se que as práticas espirituais, incluindo as religiosas, servem como forma de suporte e enfrentamento diante da doença do familiar. A espiritualidade contribui para interpretar a doença dando um sentido e um significado a ela. Em relação à vivência espiritual de pessoas com condições de saúde comprometidas, ganham destaque os estudos sobre o processo de morte e morrer. A qualidade de vida dos profissionais de saúde depende também de sua espiritualidade, principalmente no que se refere a um melhor enfrentamento do estresse cotidiano na atuação profissional.

Tendo como limite deste estudo apenas publicações das quais participavam enfermeiros, torna-se necessário o desenvolvimento de mais pesquisas abarcando os demais profissionais da área da saúde, de modo a contribuir para a construção de um corpo de conhecimento acerca da espiritualidade neste campo. Sendo uma dimensão do ser humano que integra as demais dimensões, torna-se evidente sua valorização como um dos eixos de cuidado ao outro contribuindo para uma prática assistencial mais humanizada e integral.

\section{REFERÊNCIAS}

1. Peres MFP, Arantes ACLQ, Lessa OS, Caous CA. A importância da integração da espiritualidade e da religiosidade no manejo da dor e dos cuidados paliativos. Rev. psiquiatr. clín. 2007;34(supl 1):82-7.

2. Vasconcelos EM. Espiritualidade na educação popular em saúde. Cad. CEDES. 2009;29(79):323-34.

3. Valle JER. Religião e espiritualidade: um olhar psicológico. In: Amatuzzi MM, (organizador). Psicologia e espiritualidade. São Paulo: Paulus; 2005.

4. Mendes KDS, Silveira RCCP, Galvão CM. Revisão Integrativa: Método de pesquisa para incorporação de evidências na saúde e na enfermagem. Texto Contexto Enferm. 2008;17(4):758-64.

5. Vila VSC, Rossi LA. A qualidade de vida na perspectiva de clientes revascularizados em reabilitação: estudo etnográfico. Rev. Latino-Am. Enfermagem. 2008;16(1):1-8.

6. Pinto C, Pais-Ribeiro JL. Construção de uma escala de avaliação da espiritualidade em contextos de saúde. Arq Med. 2007;21(2):47-53.

7. Cavendish R, Luise BK, Bauer M, Gallo MA, Horne $\mathrm{K}$, Medefindt $\mathrm{J}$ et al. Recognizing opportunities for spiritual enhancement in young adults. Nurs Diagn. 2001;12(3):77-91.

8. Silva MG, Boemer MR. La vivencia de envejecer en la perspectiva fenomenológica. Rev. Latino-Am. Enfermagem. 2009;17(3):380-6.

9. Fernandes MGM, Silva AO, Neves LLSN, Medeiros ACT. Indicadores e condições associadas ao envelhecimento bem-sucedido: revisão integrativa da literatura. Cogitare enferm. 2011;16(3):543-8.

10. Elias ACA, Giglio JS, Pimenta CAM. Análise da natureza da dor espiritual apresentada por pacientes terminais e o processo de sua re-significação através da intervenção relaxamento, imagens mentais e espiritualidade (RIME). Rev. Latino-Am. Enfermagem. 2008;16(6):1-8.

11. Elias ACA, Giglio JS, Pimenta CAM, El-Dash LGY. Programa de treinamento sobre a intervenção terapêutica "relaxamento, imagens mentais e espiritualidade” (RIME) para re-significar a dor espiritual de pacientes terminais. Rev. psiquiatr. clín. 2007;34(supl 1):60-72. 
12. Silva EP, Sudigursky D. Concepções sobre cuidados paliativos: revisão bibliográfica. Acta Paul. Enferm. 2008;21(3):504-8.

13. Souza S. O vivido pelo cliente em pré-operatório de cirurgia cardíaca. Online Braz J Nurs. [Internet] 2006;5(2) [acesso em 31 mar 2011]. Disponível: http:// www.objnursing.uff.br//index.php/nursing/article/ view/403/97

14. Nunes AA, Rodrigues BSC, Soares EM, Soares S, Miranzi SSC. Qualidade de vida de pacientes hemofílicos acompanhados em ambulatório de hematologia. Rev. Bras. Hematol. Hemoter. 2009;31(6):1-7.

15. Reis MG, Costa IP. Qualidade de vida relacionada à saúde em pacientes com lúpus eritematoso sistêmico no Centro-Oeste do Brasil. Rev. Bras. Reumatol. 2010;50(4):408-22.

16. Gaspar J, Reis RK, Pereira FMV, Neves LAS, Castrighini CC, Gir E. Qualidade de vida de mulheres vivendo com o HIV/Aids em município do interior paulista. Rev Esc Enferm USP. 2011;45(1):230-6.

17. Abu-Saad HH, Abboud S. Health-related quality of life among breast cancer patients in Lebanon. Eur J Oncol Nurs. 2012;16(5):491-7.

18. Gonçalves MAS, Pillon SC. Adaptação transcultural e avaliação da consistência interna da versão em português da Spirituality Self Rating Scale. Rev. psiquatr. clín. 2009;36(1):10-5.

19. Pillon SC, Santos MA, Gonçalves AMS, Araújo KM. Uso de álcool e espiritualidade entre estudantes de enfermagem. Rev Esc Enferm USP. 2011;45(1):100-7.

20. BackesDS,BackesMS, MedeirosHMF, SiqueiraDF, Pereira $\mathrm{SB}$, Dalcin CB et al. Oficinas de espiritualidade: alternativa de cuidado para o tratamento integral de dependentes químicos. Rev Esc Enferm USP 2012;46(5):1254-9.

21. Caixeta CR, Nascimento LC, Pedro IC, Rocha SM. Spiritual support for people living with HIV/Aids: A Brazilian explorative, descriptive study. Nurs Health Sci. 2012;14(4):514-9.

22. Molzahn A, Sheilds L, Bruce A, Stajduhar K, Makaroff KS, Beuthin R et al. People living with serious illness: stories of spirituality. J Clin Nurs. 2012;21(15-16):2347-56.

23. Chaves ECL, Carvalho EC, Beijo LA, Goyatá SLT, Pillon SC. Eficácia de diferentes instrumentos para a atribuição do diagnóstico de enfermagem Sofrimento espiritual. Rev. Latino-Am. Enfermagem [Internet] 2011;19(4) [acesso em 20 dez 2012]. Disponível: http:// dx.doi.org/10.1590/S0104-11692011000400008

24. Guerrero GP, Zago MMF, Sawada NO, Pinto MH. Relação entre espiritualidade e câncer: perspectiva do paciente. Rev Bras Enferm. 2011;64(1):53-9.

25. Chaves ECL, Carvalho EC, Terra FS, Souza L. Validação clínica de espiritualidade prejudicada em pacientes com doença renal crônica. Rev. Latino-Am. Enfermagem [Internet] 2010;18(3) [acesso em 31 mar 2011]. Disponível: http://dx.doi.org/10.1590/S010411692010000300003

26. Yang CT, Narayanasamy A, Chang SL. Transcultural spirituality: the spiritual journey of hospitalized patients with schizophrenia in Taiwan. J Adv Nurs. 2012;68(2):358-67.

27. Araújo MAM. Sentido da vida, espiritualidade e sociopoética: convergências para a produção de novos conhecimentos e para o cuidado clínico. InterfaceComunic., Saude, Educ. 2009;13(28):245-6.

28. Amaral MTMP. Encontrar um novo sentido da vida: um estudo explicativo da adaptação após lesão medular. Rev Esc Enferm USP. 2009;43(3):573-80.

29. Penha RM, Silva MJP. Do sensível ao Inteligível: novos rumos comunicacionais em saúde por meio do estudo da Teoria Quântica. Rev Esc Enferm USP. 2009;43(1):208-14.

30. Penha RM, Silva MJP. Significado de espiritualidade para a enfermagem em cuidados intensivos. Texto Contexto Enferm. 2012;21(2): 260-8.

31. Hayes L, Aggar C, Verde J, Carey M. Spirituality and spiritual caring: nurse' perspectives and practice in palliative and acute care environments. J. Clin. Nurs. 2012;21(15-16):2126-35.

32. TomassoCS,BeltrameIL,LucchettiG.Conhecimentoseatitudes dedocentesealunosemenfermagemnainterfaceespiritualidade, religiosidade e saúde. Rev. Latino-Am. Enfermagem [Internet] 2011;19(5) [acesso em 20 dez 2012]. Disponível: http://dx.doi.org/10.1590/S0104-11692011000500019

33. Ferrell BR, Baird P. Deriving Meaning and Faith in Caregiving. Semin Oncol Nurs. 2012;28(4):256-61.

34. Fogaça MC, Carvalho WB, Nogueira-Martins LA. Estudo preliminar sobre a qualidade de vida de médicos e enfermeiros intensivistas pediátricos e neonatais. Rev Esc Enferm USP. 2010;44(3):708-12. 
35. Benko MA, Silva MJP. Pensando a espiritualidade no ensino de graduação. Rev. Latino-Am. Enfermagem. 1996;4(1):71-85.

36. Gussi MA, Dytz JLG. Religião e espiritualidade no ensino e assistência de enfermagem. Rev. bras. enferm. 2008;61(3):377-84.

37. Connell MT. Spirituality and spiritual care from a Careful Nursing perspective. J Nurs Manag. 2012;20(8):900-1001.

38. Gutierrez BAO, Ciampone MHT. O processo de morrer e a morte no enfoque dos profissionais de enfermagem de UTIs. Rev Esc Enferm USP. 2007;41(4):660-7.

39. Mendes IAC, Trevizan MA, Ferraz CA, Fávero N. The re-humanization of the executive nurse's job: a focus on spiritual dimension. Rev. Latino-Am. Enfermagem. 2002;10(3):401-7.

40. Guido LA, Linch GFC, Andolhe R, Conegatto CC, Tonini CC. Estressores na assistência de enfermagem ao potencial doador de órgãos. Rev. Latino-Am. Enfermagem. 2009;17(6):1-7.

41. Hsiao YC, Wu HF, Chien LY, Chiang CM, Hung YH, $\mathrm{PH}$ Peng. The differences in spiritual health between non-depressed and depressed nurses. J. Clin. Nurs. 2012;21(11-12):1736-45.

42. Dezorzi LW, Crossetti MGO. A espiritualidade no cuidado de si para profissionais de enfermagem em terapia intensiva. Rev. Latino-Am. Enfermagem. 2008;16(2):1-7.

43. Nascimento LC, Oliveira FCS, Moreno MF, Silva FM. Cuidado espiritual: componente essencial da prática da enfermeira pediátrica na oncologia. Acta Paul. Enferm. 2010;23(3):437-40.

44. Véras RM, Vieira JMF, Morais FRR. A maternidade prematura: o suporte emocional através da fé e religiosidade. Psicol. estud. 2010;15(2):325-32.

45. Alarcão ACJ, Carvalho MDB, Pelloso SM. A morte de um filho jovem em circunstância violenta: compreendendo a vivência da mãe. Rev. Latino-Am. Enfermagem. 2008;16(3):1-7.

46. Mendonça FF, Garanhani ML, Martins VL. Cuidador familiar de sequelados de acidente vascular cerebral: significado e implicações. Physis. 2008;18(1):143-58.

47. Pizzignacco TP, Mello DF, Lima RG. A experiência da doença na fibrose cística: caminhos para o cuidado integral. Rev Esc Enferm USP. 2011;45(3):638-44.
48. Brondani CM, Beuter M, Alvim NAT, Szareski C, Rocha LS. Cuidadores e estratégias no cuidado ao doente na internação domiciliar. Texto Contexto Enferm. 2010;19(3):504-10.

49. Rocha MPF, Vieira MA, Sena RR. Desvelando o cotidiano dos cuidadores informais de idosos. Rev Bras Enferm. 2008;61(6):801-8.

50. Leite MF, Collet N, Gomes IP, Kumamoto LHMCC. Enfrentamento da condição crônica na infância pelo cuidador familiar: pesquisa qualitativa. Online Braz J Nurs [Internet] 2010;9(3) [acesso em 31 mar 2011]. Disponível: http://www.objnursing.uff.br/index.php/ nursing/article/view/j.1676-4285.2010.3147/html_1

51. Paula ES, Nascimento LC, Rocha SMM. Religião e espiritualidade: experiência de famílias de crianças com insuficiência renal crônica. Rev Bras Enferm. 2009;62(1):100-6.

52. Tomeleri KR, Marcon SS. Práticas populares de mães adolescentes no cuidado aos filhos. Acta Paul. Enferm. 2009;22(3):272-80.

53. Lima AB, Rosa DOS. The life meaning of the parents of the critical patient. Rev Esc Enferm USP. 2008;42(3):539-45.

Cogitare Enferm. 2013 Abr/Jun; 18(2):372-8 